\title{
The coupling of optimal economic growth and climate dynamics
}

\author{
Olivier Bahn · Laurent Drouet • Neil R. Edwards • \\ Alain Haurie · Reto Knutti · Socrates Kypreos • \\ Thomas F. Stocker $\cdot$ Jean-Philippe Vial
}

Received: 11 October 2004 / Accepted: 14 February 2006 / Published online: 27 October 2006

(C) Springer Science + Business Media B.V. 2006

\begin{abstract}
In this paper, we study optimal economic growth programs coupled with climate change dynamics. The study is based on models derived from MERGE, a well established integrated assessment model (IAM). We discuss first the introduction in MERGE of a set of "tolerable window" constraints which limit both the temperature change and the rate of temperature change. These constraints, obtained from ensemble simulations performed with the Bern 2.5-D climate model, allow us to identity a domain intended to preserve the Atlantic thermohaline circulation. Next, we report on experiments where a two-way coupling is realized between the economic module of MERGE and an intermediate complexity "3-D-" climate model (C-GOLDSTEIN) which computes the changes in climate and mean temperature. The coupling is achieved through the implementation of an advanced "oracle based
\end{abstract}

O. Bahn

GERAD and MQG, HEC Montréal, Montréal (Qc), H3T 2A7, Canada

e-mail: olivier.bahn@hec.ca

L. Drouet · A. Haurie · J.-P. Vial

LOGILAB-HEC, University of Geneva, 1211 Geneva, Switzerland

e-mail: laurent.drouet@hec.unige.ch

e-mail: alain.haurie@hec.unige.ch

e-mail: jean-philippe.vial@hec.unige.ch

N. R. Edwards

Earth Sciences, CEPSAR, Open University, Milton Keynes, MK7 6AA, UK

e-mail: n.r.edwards@open.ac.uk

R. Knutti

National Center for Atmospheric Research, Boulder, CO 80305, USA

e-mail: knutti@ucar.edu

S. Kypreos

Paul Scherrer Institute, 5232 Villigen, Switzerland

e-mail: socrates.kypreos@psi.ch

T. F. Stocker

Climate and Environmental Physics, Physics Institute, University of Bern, 3012 Bern, Switzerland

e-mail: stocker@ climate.unibe.ch 
optimization technique" which permits the integration of information coming from the climate model during the search for the optimal economic growth path. Both cost-effectiveness and cost-benefit analysis modes are explored with this combined "meta-model" which we refer to as GOLDMERGE. Some perspectives on future implementations of these approaches in the context of "collaborative" or "community" integrated assessment modules are derived from the comparison of the different approaches.

\section{Introduction}

The building of integrated assessment models of climate change has followed two main approaches. In one strand of research, we find highly aggregated and integrated models where the economy, the climate system and the damage evaluation form components of a modular dynamical system. The integrated climate-economy system behavior is then simulated over a long time horizon, for specified economic policies. An optimization technique is used to identify a cost-effective or an efficient global climate policy. The archetypal models having this structure are the DICE family of models (Nordhaus, 1993; Nordhaus and Boyer, 2000), MERGE (Manne et al. 1995), and more recently ICLIPS (Toth et al. 2003), among many others. In all these models the description of climate dynamics is highly simplified and reduces to a relatively small number of difference equations describing the carbon cycle, the greenhouse gas forcing and the resulting average surface air temperature. Another strand of integrated assessment modeling effort is typically represented by the MIT integrated global system model (IGSM) (Prinn et al. 1999) where the assessment tool is composed of loosely interconnected modules, such as a detailed multi-region computable general equilibrium model to describe the world economy and a high-resolution general circulation model to describe the climate dynamics. There is no hard-linking between the different modules. In such a system, each module is run more or less independently with boundary conditions that are specified by taking into account the results of simulations performed on the other models composing the system. The coupling between these heterogeneous classes of models is therefore a challenge which has been recognized by the Collaborative Integrated Assessment Model $\left(\right.$ CIAM $\left.^{n}\right)$ research framework (Jaeger et al. 2002) and first tackled explicitly by Leimbach and Jaeger (2004).

The aim of this paper is to present methods that permit the integration into a relatively detailed optimal economic growth model of information concerning climate change that is provided by a relatively detailed, spatially resolved climate model. A guiding objective in the research reported here has been to combine the methodological elegance of highly integrated models with the sectoral relevance of systems combining state-of-the-art models of climate and economics. The economic model that we use follows the Ramsey-Solow longterm optimal economic growth paradigm and forms part of MERGE, a well established IAM developed to study the economic dimension of climate change. On the climate side we use (i) the Bern 2.5-D model (Stocker et al. 1992; Stocker and Schmittner 1997) which is well known in the climate community and (ii) C-GOLDSTEIN (Edwards and Marsh 2005) which is a novel higher dimensional intermediate complexity model lending itself to relatively fast simulations of climate histories. In the first approach the Bern 2.5-D model is used to propose a combined constraint on temperature change and its rate of change to attempt to avoid a breakdown of the Atlantic thermohaline circulation (THC). This constraint is added to MERGE which is then run in a cost-effectiveness mode. In a sense, the detailed climate model is used to identify a "tolerable window" (TW) constraint that is introduced in the IAM. The second approach is more ambitious as it establishes a direct dialogue between two specialized 
modules: the first module is concerned with the economic growth dynamics, the accumulation of GHGs (carbon cycle dynamics) and the damage due to climate change; the other module deals with the climate dynamics per se. A cost-effectiveness and a cost-benefit analysis are thus conducted by implementing an "oracle-based" optimization technique that establishes a coordination between these two modules. This direct coupling between detailed economic and climate models has been made possible by two scientific advances: (a) the building of computationally efficient intermediate complexity climate models that lend themselves to fast simulations, and (b) the development of oracle-based optimization techniques that converge in relatively few calls to the oracles. The implementation of such a combination of advanced optimization and climate modeling techniques opens new avenues in the development of IAMs and will thus be presented in some detail.

The remainder of the paper is organized into three parts. In the first part, we review the structure of MERGE. In the second part, we address the issue of constraining the economic development path through climate change limits that are intended to avoid the occurrence of a threshold event, namely the collapse of the THC. These constraints are identified via an ensemble simulation conducted with the Bern 2.5-D climate model. In the third part, we report on experiments where the economic growth module of MERGE is directly coupled with a higher dimensional climate model with fully 3-D ocean dynamics. In conclusion we discuss possible further applications and extensions of the approach.

\section{The MERGE modeling framework}

The Model for Evaluating the Regional and Global Effects of greenhouse gas reduction policies (MERGE) was introduced by Manne et al. (1995). MERGE comprises nine geopolitical regions and four modules (energy, macroeconomic, climate and damage modules) as displayed in Figure 1.

The energy module is a bottom-up process model. It describes the energy supply sector of each region, in particular the generation of electricity and the production of non-electric energy. It captures price-dependent substitutions of energy forms and energy technologies to comply with greenhouse gas (GHG) emission abatements. The macroeconomic module is a top-down macroeconomic growth model. It balances the rest of the economy of a given region using a nested constant elasticity of substitution production function. It captures

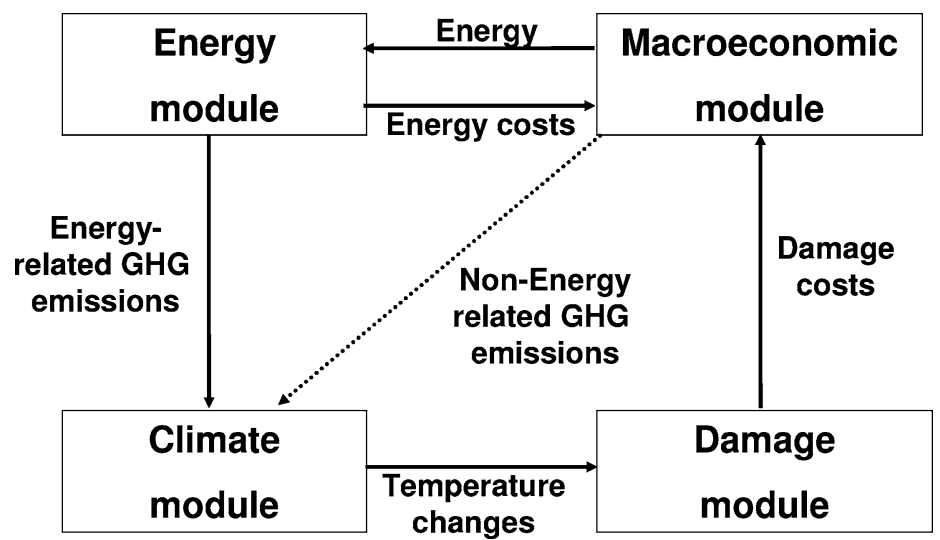

Fig. 1 Overview of the MERGE modules. 
macroeconomic feedbacks between the energy system and the rest of the economy, for instance impacts of higher energy prices on economic activities. The regional models yield anthropogenic emissions of $\mathrm{CO}_{2}, \mathrm{CH}_{4}$ and $\mathrm{N}_{2} \mathrm{O}$. The climate module computes changes in atmospheric GHG concentrations, impacts on the Earth's radiative forcing balance and finally mean surface air temperature (SAT) changes. Finally, the damage module assesses how temperature changes cause commensurate economic losses, distinguishing among market damages (damages that can be valued using market prices) and non-market damages (e.g. damages caused to biodiversity that do not have direct market values). A discussion of the evaluation of climate change damage in MERGE is given by Manne and Richels (2005).

The mathematical formulation of the regional energy-economy modules is an optimization problem, where the economic equilibrium is determined by a single optimization. More precisely, the model maximizes a welfare function defined as the net present value of the logarithm of regional consumption (adjusted for non-market damages when following a cost-benefit approach). It aggregates the regional welfare functions into a global welfare function using Negishi weights (Negishi 1972). The regional submodels are further linked by international trade of oil, gas, synthetic fuels, industrial-energy-intensive products, emission permits, and an aggregate good expressed in a monetary unit that represents all other (nonenergy) traded goods. A global constraint ensures that international trade is balanced.

We use here version 4.6 of MERGE in which the climate module parameters have been revised in accordance with the findings of the IPCC as described by Bahn et al. (2004). Uncertainty in climate-module response is reduced to uncertainty in two key parameters, the basic atmospheric sensitivity to radiative forcing (the equilibrium temperature change for a doubling of $\mathrm{CO}_{2}$ above pre-industrial level) and a timescale for the lag of the atmospheric temperature behind its equilibrium value, principally as a result of heat uptake by the ocean. In the case studies we present, the climate sensitivity ranges between 2 and $4{ }^{\circ} \mathrm{C}$ in accordance with IPCC (2001b), whereas the lag time is set to 60 years, a mean value obtained from an ensemble of 1000 runs of C-GOLDSTEIN (Edwards and Marsh 2005). Notice also that, for the sake of simplicity, the model version we use does not consider endogenous technological progress in the energy sector.

\section{Tolerable window constraints derived from the Bern 2.5-D climate model}

In this section, we introduce tolerable window constraints derived from the Bern 2.5-D climate model. The German Advisory Council on Climate Change (WBGU) ${ }^{1}$ proposed to limit temperature increase (relative to pre-industrial levels) by $2{ }^{\circ} \mathrm{C}$ and rate of temperature increase by $0.2^{\circ} \mathrm{C}$ per decade (WBGU 2003). This position has recently been echoed by the European Council. ${ }^{2}$ In this section, rather than using subjectively defined values we introduce objectively defined constraints limiting the probability of a THC collapse. These constraints are designed using an ensemble of simulations performed with the Bern 2.5-D model.

\subsection{TW constraints to preserve the ATLANTIC THC}

The imposition of TW constraints designed to preserve the Atlantic THC has been considered by several authors, (Bahn et al. 2004; Bruckner and Zickfeld 2004; Keller et al. 2004). We

\footnotetext{
${ }^{1}$ Wissenschaftlicher Beirat der Bundesregierung Globale Umweltveränderungen.

${ }^{2}$ EU Council 7631/04.

Springer
} 
follow here the approach of Bahn et al. (2004) (henceforth BEKS) who have proposed simple climate constraints designed to prevent the collapse of the THC. We shall describe briefly how these constraints are obtained and present new numerical results where uncertainty is explicitly taken into account in the decision-making process.

To estimate the level and rate of GHG emissions likely to induce a collapse of the THC, BEKS used the Bern 2.5-D climate model which is based on 2-dimensional (latitude-depth) representations of the flow in each of the Pacific, Atlantic and Indian Oceans, connected via a 2-dimensional (longitude-depth) representation of the Southern Ocean (Stocker et al. 1992). The model also includes a 1-layer energy and moisture balance representation of the atmosphere (Schmittner and Stocker 1999) and a thermodynamic representation of sea ice. In the version used here, GHG forcing is parameterized as a change in radiative forcing at the top of the atmosphere.

Constraints were derived from an ensemble of 25000 Monte-Carlo simulations with values of climate sensitivity and GHG concentrations chosen randomly within specified bounds but with future emissions scaled to SRES scenario Bl (very similar values for the TW constraints were obtained using SRES A2). Only simulations which matched observed global mean surface warming from 1900 to 2000 and observed ocean heat uptake from 1955 to 1995 were retained, around $10 \%$ of the total number; for details see Knutti et al. (2003). This set was used to derive approximate conditions for a collapse of the THC, defined here as a decrease of over $50 \%$ in the maximum integrated northward water-mass flux in the Atlantic. Note that since the circulation has a qualitatively bimodal behaviour (Knutti and Stocker 2002), our choice of $50 \%$ for the threshold value should have little effect on the results. As found by Stocker and Schmittner (1997) both the maximum absolute warming and the maximum warming rate need to be considered, although the sensitivity of the THC to warming rate is not found in all models (Lenton et al. 2005). BEKS thus applied linear constraints to these two quantities as follows: the limit on absolute warming increases linearly between $0.70{ }^{\circ} \mathrm{C}$ in 2000 and $1.42^{\circ} \mathrm{C}$ in 2100 , whereas the limit on warming rate decreases linearly between $0.24^{\circ} \mathrm{C}$ in 2000 and $0.13^{\circ} \mathrm{C}$ in 2100 .

\subsection{Numerical results}

Using the version of MERGE described in section 2, BEKS analysed climate policies preserving the Atlantic THC, namely policies that impose constraints (as defined above) on maximum absolute warming and maximum warming rate. These policies (denoted $\mathrm{P}^{*}$ ) were contrasted with a business-as-usual scenario, without any GHG emission control, and a Kyoto-like GHG reduction policy. Of specific importance for these scenarios is the uncertainty in the response of the physical climate system (encapsulated here in the climate sensitivity). This uncertainty can be addressed using a scenario-by-scenario analysis: separately considering several cases where all parameters are perfectly known (the perfect foresight situation), for example, a case denoted *L with "low" climate sensitivity $\left(2^{\circ} \mathrm{C}\right)$ and a case denoted ${ }^{*} \mathrm{H}$ with "high" 3 climate sensitivity $\left(4^{\circ} \mathrm{C}\right)$. This scenario-by-scenario approach has been used by BEKS. In the present paper we deal with the uncertainty characterizing the climate sensitivity by implementing a "stochastic programming" approach that considers simultaneously the different scenarios for the uncertain climate parameter without the assumption of perfect foresight. We assume

\footnotetext{
${ }^{3}$ With respect to the current uncertainty range proposed by (IPCC, $\left.2001 \mathrm{~b}\right)\left(1.5\right.$ to $\left.4.5^{\circ} \mathrm{C}\right)$. It is, however, worth noticing that our high case is not a worst case scenario, as new studies (Knutti et al. 2002; Stainforth et al. 2005; Knutti et al. 2006; Stott et al. 2006) indicate that the climate sensitivity range might be higher.
} 


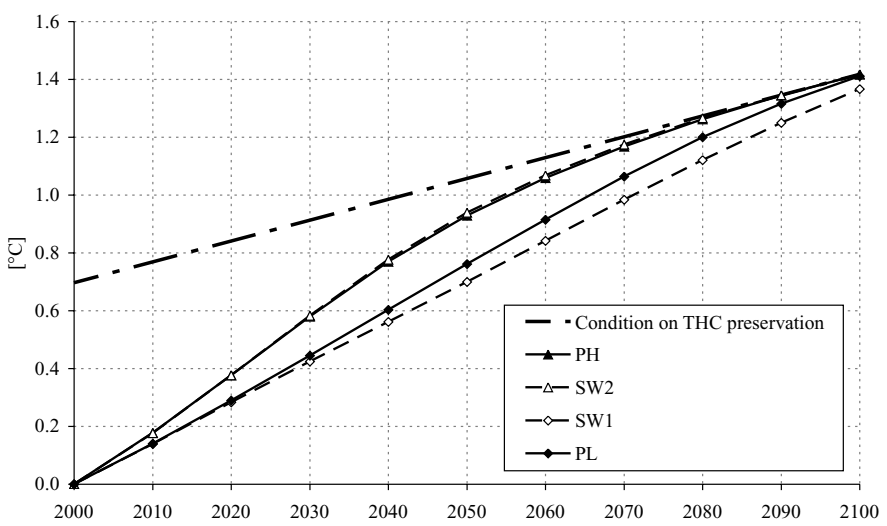

Fig. 2 Warming (from 2000) for the THC preservation scenarios (denoted PL and PH in the scenario-byscenario approach, and SW1 and SW2 in the stochastic programming approach), as well as necessary condition for preserving the THC in terms of maximum warming. Cases PL and SW1 (resp. PH and SW2) correspond to low (resp. high) climate sensitivity assumptions.

a particular future date $(2030)^{4}$ at which a perfect knowledge of climate sensitivity will be obtained. The stochastic process describing the unfolding of uncertainty is then represented by a two-stage event tree. This consists of a first stage, or trunk, that describes the measures (e.g., GHG abatement) to be implemented when climate sensitivity is still uncertain (between 2000-2030), and a second stage, or branches, that describe recourses (i.e., revised GHG abatement levels) to be implemented once perfect knowledge is obtained. Branches correspond to alternative settings for the uncertain climate parameter (corresponding to cases ${ }^{*} \mathrm{~L}$ and ${ }^{*} \mathrm{H}$ ) that define two states of the world (SW1 and SW2, respectively). Following the PDF generated by the Bern 2.5-D model, the probabilities used are: $20 \%$ for SW1 and $80 \%$ for SW2. We will also examine the effect of alternative probability distributions.

We assume also that there is a social planner (world regulator) that monitors the Earth's warming and is able to impose worldwide GHG emission reductions such that the necessary conditions on THC preservation are respected. Figure 2 displays the evolution of the warming 5 which constitutes the more demanding condition for the THC preservation. For the scenario SW1, the THC preservation constraint becomes binding by 2120 (the model's horizon).

The stochastic approach proposes to policy makers a "hedging" climate policy. ${ }^{6}$ This corresponds to a single trajectory for GHG emissions up to the date at which uncertainty is resolved (here, 2030). Following this trajectory corresponds to a "least regret" strategy that balances present regret of imposing premature and costly emission reduction (when climate sensitivity is low) with future regret of neglected reduction in the past (when climate sensitivity is high). This strategy is defined by maximizing the sum of the welfare of the two states of the world weighted by their respective probability of occurrence. Figure 3 displays the resulting world energy-related $\mathrm{CO}_{2}$ emissions under the different THC preservation scenarios.

\footnotetext{
${ }^{4}$ In contrast to several other studies such as Keller et al. (2004) which assume a longer learning time.

${ }^{5}$ Between 2000 and 2030, the computation of the warming in the SW1 (resp. SW2) case uses the emissions of the hedging path (see below) and assumes a low (resp. high) climate sensitivity.

${ }^{6}$ See for instance F.L. Toth and M. Mwandosya, "Decision-making Frameworks", pp. 612-614, in (IPCC, 2001a). 


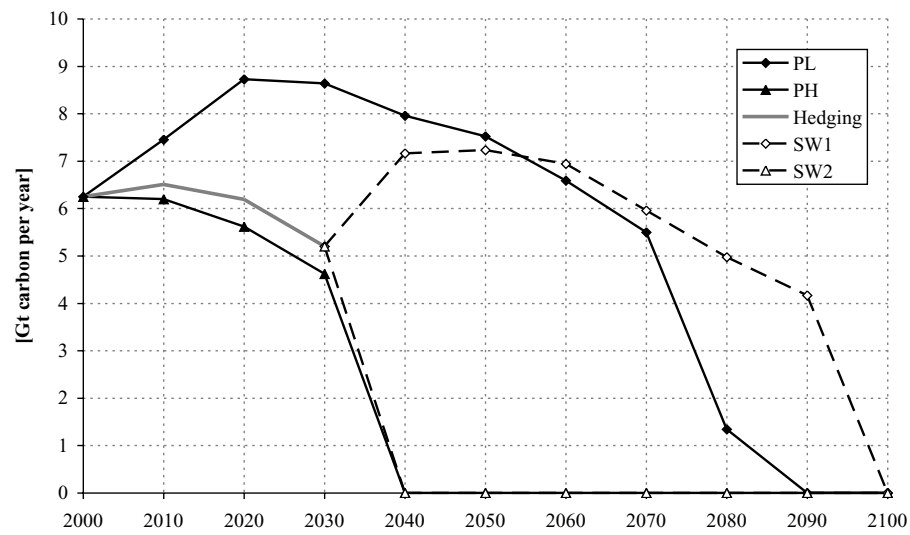

Fig. 3 World energy-related $\mathrm{CO}_{2}$ emission trajectories under the THC preservation scenarios (denoted PL and $\mathrm{PH}$ in the scenario-by-scenario approach; and Hedging, SW1 and SW2 in the stochastic programming approach). Cases PL and SW1 (resp. PH and SW2) correspond to low (resp. high) climate sensitivity assumptions.

Figure 3 shows, for the stochastic programming approach, a unique emission path between 2000 and 2030 (before the resolution of climate uncertainties), whichever state is finally realized (SW1 or SW2). This corresponds to the hedging strategy which, in this case, closely follows the PH emission path. After 2030, once the true climate sensitivity is known, the stochastic programming approach also proposes an optimal path to adapt to the assumed true state. In the low climate sensitivity case (SW1), emissions are allowed to increase until 2050 (to compensate for early reductions) and are reduced afterwards, albeit less quickly than in the PL emission path. In the high climate sensitivity case (SW2), emissions are required to reduce to zero as early as 2040 , as in the $\mathrm{PH}$ emission path. Such a drastic abatement can only be more severe when assuming a climate sensitivity higher than $4{ }^{\circ} \mathrm{C}$, a possibility that has been recognized in recent studies. The fact that the hedging path follows the PH emission trajectory is not simply a result of the high probability associated with SW2. To demonstrate this we have conducted a sensitivity analysis, assuming alternative probability settings for the two states of the world (SW1 and SW2, respectively): (50\%, 50\%), (70\%, 30\%) and (90\%, 10\%). Emissions in 2030 are respectively 5.3, 5.4 and $5.5 \mathrm{Gt} \mathrm{C}$, very close to the value of $5.2 \mathrm{Gt} \mathrm{C}$ found when assuming a $(20 \%, 80 \%)$ probability distribution. This is explained by the fact that one must be able to reduce emissions to zero by 2040 when climate sensitivity is revealed to be high. In a sense, the stochastic programming approach here is close to a maximin approach, where the social planner's decisions (levels of abatement) are driven by the consideration of the worst possible outcome (high climate sensitivity).

This example has demonstrated that TW constraints based on information obtained from more advanced climate models can be included in an economic growth model coupled with a simple climate model (in this case the integrated assessment model MERGE). However, the temperature change (in MERGE) is still predicted by a "zero-dimensional" climate module. There is therefore a lack of consistency between the model (Bern 2.5-D) that is used to delineate the TW constraints and the climate model (within MERGE) used to simulate the climate-economy feedbacks. In the next section we consider how this situation can be improved by allowing a two-way exchange of information between economic and climate models, so that the two models share a consistent representation of climate change. 


\section{An oracle-based optimization approach for the coupling of MERGE with the C-GOLDSTEIN climate model}

In this section we describe a general method permitting a coupling between independent and specialized models in order to build an IAM. The method exploits a recently developed mathematical programming method, the "Oracle Based Optimization Technique" (OBOT), that permits a coherent dialogue between models. In the implementation described here, one module gives a description of the world economy and carbon-cycle dynamics and is obtained from MERGE. A second module is a 3-D climate model of reduced complexity. The result is an all-encompassing model with an economic sub-model, a climate sub-model and a master program which controls the optimization procedure through a consistent dialogue between the models.

In previous experiments with this approach reported by Drouet et al. (2005a,b) a coupling has been realized between the economic part of the DICE model and the C-GOLDSTEIN climate model. The implementation of OBOT described here for the coupling of MERGE with C-GOLDSTEIN represents a significant advance. Not only is MERGE a far more comprehensive and detailed model of the global energy economy than DICE but furthermore, by coupling climate and economic models both of which have some spatial resolution, we have made an important step towards an IAM with spatially resolved feedback between climate and economy. In the remainder of this section we first briefly recall the main features of the C-GOLDSTEIN model, then present OBOT, the two coupling modes, namely costeffectiveness (CE) and cost-benefit (CB) and finally the results obtained from this coupling.

\subsection{The C-GOLDSTEIN climate model}

C-GOLDSTEIN is a flexible geometry, efficient, frictional geostrophic, 3-D global ocean model with eddy-induced and isopycnal mixing coupled with an energy and moisture balance atmosphere and a dynamic and thermodynamic sea-ice component. The model dynamics and behaviour are described briefly by Drouet et al. (2005a), and more fully by Drouet et al. (2005b). With an integration speed of one or two thousand years per hour on a modern PC (Pentium-IV, 2.4 GHz), it is an order of magnitude less efficient than the Bern 2.5-D model, but 3 or 4 orders of magnitude faster than conventional, high-resolution models such as HadCM3 (Gordon et al. 2000) and 1 or 2 orders of magnitude faster than other intermediate complexity models such as ECBILT-CLIO (Haarsma et al. 2001) or the UVic model (Weaver et al. 2001), which has a very similar atmosphere. This efficiency is mainly a result of low spatial resolution and simplified dynamics. For the studies described here, the ocean component is configured with 8 vertical levels and 36 by 36 cells in the horizontal. Gross features of the global-scale ocean circulation and climate can be reasonably well represented, particularly when model parameters are automatically calibrated with respect to observational data by performing large numbers of simulations (Hargreaves et al. 2005). For the experiments reported here, however, we use default values for model parameters since our focus is on the demonstration of the coupling technique. All components share the same horizontal grid, but the model atmosphere has only one vertical level, so that atmospheric processes are represented by a vertical balance of energy and moisture plus simple horizontal transport by anisotropic diffusion and fixed advection by the wind. Atmospheric radiative forcing is parameterised by a polynomial function of temperature and humidity with a greenhouse gas forcing term proportional to the $\log$ of the ratio of $\mathrm{CO}_{2}$ concentration compared to its pre-industrial value. Feedbacks involving changes in atmospheric circulation and precipitation patterns, and feedbacks involving the land surface, are relatively poorly represented or ignored. Sea-ice 
height and areal coverage are similarly governed by a vertical heat and freshwater balance, plus advection by surface currents with a diffusive term to represent unresolved processes. Given these dynamical simplifications, the model is most suitable for studies of large-scale ocean circulation and long-term climate change, and for probabilistic climate-change studies and integrated assessment modelling. The model forms a component of the Grid Enabled Integrated Earth System Model (GENIE) project ${ }^{7}$ in the context of which an Earth System Model with more detailed representations of atmosphere, land-ice, ocean biogeochemistry and land-surface processes is under development.

For the experiments reported here temporal variability in solar forcing is ignored and the model approaches an equilibrium state, after a few thousand years of integration, which is essentially independent of the initial conditions and can be taken to represent the preindustrial climate. The model is then integrated forwards with observed atmospheric $\mathrm{CO}_{2}$ concentrations from 1795 to 1995 to produce an initial condition for the coupled runs.

\subsection{The oracle based optimization technique}

The optimization of large-scale systems through decomposition techniques has given rise to important developments related to non-differentiable optimization, column generation and cutting plane techniques. The version of an Oracle Based Optimization Technique (OBOT) that we use was first proposed by Goffin et al. (1992). Since then several improvements and implementation advances have been made as described by Péton and Vial (2001) and Babonneau et al. (2005).

In the world of convex optimization, one searches for the minimum of a convex function over a convex set of admissible values for the variables. OBOT converges to the optimum by sending a sequence of query points which are proposed values for the decision variables. If the query point is not feasible (that is, it violates the imposed constraints) an "oracle" sends back information in the form of a separating plane which places the query point in one half-space and the admissible set in the other. If the query point is feasible, another "oracle" sends back information in the form of the value reached by the optimization criterion and the gradient of this function at the query point (which defines a supporting hyperplane for the epigraph of the function). From this sequence of "cutting planes" one is able to construct a polyhedral approximation of a so-called "localization set" which always contains the optimal values for the variables and the criterion function. Among possible strategies, one can choose the query point at the "center" of the current localization set. The localization set then shrinks rapidly and it has been proved that the optimum will be found with the desired level of accuracy in a manageable computational time.

The method that we implement is called Analytic Center Cutting Plane Method (ACCPM) in reference to the way one defines the center where one selects the query point. In this method, the criterion and the constraints do not have to be explicitly defined. An implicit definition permitting a computation of a value of the function at a query point and an evaluation of its gradient at this point will suffice. It is this feature which makes the approach very attractive to couple models pertaining to different spheres.

The convergence of ACCPM towards the global optimum, as well as its rate of convergence, has been established for convex optimization problems only. In the present application, the economic model is convex, but we have no guarantee that the climate model shares this property. However, once the two models have found compatible, feasible climate and

\footnotetext{
${ }^{7}$ See http://www.genie.ac.uk
} 
emission variables, we can assert that the objective value of the economic model is attainable and provides an upper bound to a global optimum. It may also improve upon alternative local optima. This is the situation that prevails at the end of the optimization process in our application.

In proving that the best point found by ACCPM is a local optimum we would face two difficulties. The first one is that the cutting planes are imprecise as they are computed by finite differences, and are thus subject to error. The second difficulty stems from the fact that sensitivity analysis in a non-convex model provides information that is only valid in a neighborhood of that point, but may be invalid farther away. To cope with this second difficulty, we have implemented a simple scheme that tests whether the cutting plane created at the current query point invalidates the current best solution. If it does, we simply shift the cutting plane to preserve feasibility of the best solution. This allows for convergence of the method. In the present case, as well as in the similar applications mentioned earlier, we very seldom encountered contradictory cutting planes.

Note that even if we could compute exact derivatives in the climate model, local optimality could only be guaranteed by discarding remote cutting planes, in the spirit of a trust-region approach (Moré, 1983; Schramm and Zowe, 1992), and proving optimality via local cutting planes only. At present, little has been done to extend cutting plane schemes to the nonconvex case. Our approach is a pragmatic substitute for a rigorous trust-region scheme, taking into account that the multiple simulations of the climate model required to calculate damage gradients are computationally expensive, ruling out the possibility of using the large numbers of query points required by other algorithms such as the differential evolutional algorithm considered by Keller et al. (2004).

\subsection{Two modes of coupling}

We give here briefly the principles of OBOT when applied to the coupling of an economic growth model with a climate model in two different modes of analysis, namely costeffectiveness (CE) and cost-benefit (CB) modes. The coupling yields a new meta-IAM which is now denoted GOLDMERGE-CE or GOLDMERGE-CB according to the coupling mode that is used. The method of solution is similar for the two modes but the information exchanged between the oracles and the optimization module differs slightly. These differences and the two reduced-order problems driven by the coupling variables are discussed in the next two subsections. More details on the technical aspects can be found in Drouet et al. (2005a) and Drouet et al. (2005b). We use the economic modules and the carbon-cycle description of MERGE version 4.6 as described in Section 2.

\subsubsection{The oracles in the cost-effectiveness mode}

In cost-effectiveness mode the coupling variables between the two modules are essentially the atmospheric $\mathrm{CO}_{2}$ concentrations, denoted as a vector $C$ with a component for each decade from 2010 to 2100 . Constraining these variables controls the "economy oracle". We thus obtain an optimal growth path which maximizes the total discounted welfare criterion and satisfies these concentration constraints. The sensitivity of the optimized criterion to variations in the $C$ constraints is provided by duality analysis. The "climate oracle" C-GOLDSTEIN, receiving, as input, the concentration schedule $C$, computes the average surface air temperature (SAT) and its decadal rate of change. The master program has to find the concentration schedule $C$ which optimizes the economy under a constraint of $2{ }^{\circ} \mathrm{C}$ on the average SAT change over the whole planning horizon and $0.2^{\circ} \mathrm{C}$ on its change over each decade. This 
corresponds to the rate of temperature change constraint proposed by the WBGU and alluded to at the beginning of section 3 . The sensitivity of the temperature changes to marginal modifications of $C$ is obtained by calling C-GOLDSTEIN several times with perturbed $C$ vectors (effectively using a finite-difference approximation to the derivative).

The reduced-order optimization problem solved by OBOT can be summarized by:

$$
\max _{C \in \mathbb{R}^{n}}\{U(C) \mid \phi(C) \leq \bar{\phi}\},
$$

where $U$ is the optimum value for the welfare criterion (actually a linear combination of the welfare of each region) in the economic growth model, $\phi$ is the rate of temperature change by decade computed by the climate module with a forcing of the concentration $C$ and $\bar{\phi}=0.2$ is the upper-bound value defined by the tolerable window constraint. Notice that satisfaction of the constraint on the rate of temperature change $\left(0.2^{\circ} \mathrm{C}\right.$ per decade $)$ implies automatically that the constraint on a global temperature change of $2{ }^{\circ} \mathrm{C}$ will be satisfied over the century.

\subsubsection{The oracles in the cost-benefit analysis mode}

In cost-benefit mode the economic damage depending on temperature changes is included in the computation of the welfare function. The coupling variables, denoted by the vector $X$, represent (i) the concentration schedule $C$ from 2010 to 2100 by decade and (ii) the decadal changes in temperature $T$ over the same horizon:

$$
X=(C, \delta T) \in \mathbb{R}^{2 n},
$$

where $n=10$ is the number of periods of the time horizon. The economy oracle MERGE receives the concentrations $C$ and the temperature changes $\delta T$ and replies to this query by sending the optimal value of the global welfare function $U(X)$ corresponding to the given constraints on concentration and the given temperature changes. In addition, the economic oracle sends back a gradient vector which is easily obtained from duality analysis in the economic model, as in the cost-effectiveness mode. The climate oracle C-GOLDSTEIN computes the SAT from the concentrations $C$ and replies to the query by the value of the "gap function" $\Theta(X)$ defined as follows:

$$
\Theta(X)=\phi(C)-\delta T
$$

where $\phi(C)$ is the SAT change from 2000 computed by the climate module with the forcing of the concentrations $C$. The jacobian matrix $\nabla \Theta(X)$ is also sent back and is equal to $\gamma=$ $(\nabla \phi(\bar{C}),-I)$ where $I$ is the identity matrix. The subgradient $\nabla \phi(\bar{C})$ is evaluated numerically by C-GOLDSTEIN by finite difference approximation. The value of the function $\Theta$ must be non-positive. Therefore OBOT solves the reduced order optimization problem which is formulated as follows:

$$
\max _{X \in \mathbb{R}^{2 n}}\{U(X) \mid \Theta(X) \leq 0\},
$$

where $U(X)$ is the optimum value for the economy model and $\Theta(X)$ is the gap function defined above. In summary, then, damages are included in the welfare function in the CB mode, in which case, the temperature changes are not explicitly constrained. On the contrary, temperature constraints are explicitly included in the CE mode. 


\subsection{GOLDMERGE results}

To compare the GOLDMERGE results with those of MERGE itself, as described in Section 2, we set the climate sensitivity parameters to a common value of $2.75^{\circ} \mathrm{C}$ and the timescale for the lag of atmospheric temperature behind its equilibrium to 60 years. Note that the forcing due to $\mathrm{CH}_{4}$ and $\mathrm{NO}_{2}$ gases is taken into account in the MERGE climate module whereas in C-GOLDSTEIN it is not. While this extra forcing effect could relatively easily be accounted for in C-GOLDSTEIN its neglect is unlikely to significantly alter our conclusions since the effect of the extra gases amounts to an SAT change of only about $0.2{ }^{\circ} \mathrm{C}$ over the whole time horizon. Results are reported separately for cost-effectiveness and cost-benefit modes which are denoted by the suffices CE and CB.

\subsubsection{GOLDMERGE-CE}

Figure $4 \mathrm{~b}$ shows the resulting $\mathrm{CO}_{2}$ concentrations at the optimum in GOLDMERGE-CE and MERGE-CE. GOLDMERGE-CE $\mathrm{CO}_{2}$ concentrations reach a level of $692 \mathrm{ppmv}$ in 2100 whereas MERGE-CE concentrations reach only $560 \mathrm{ppmv}$. Figure $4 \mathrm{a}$ reveals the world $\mathrm{CO}_{2}$ energy-related emissions. These are strongly related to the $\mathrm{CO}_{2}$ concentration levels since they are computed by the MERGE energy module. Emissions follow roughly the same path during the first periods, then MERGE-CE $\mathrm{CO}_{2}$ emissions start to decrease in 2050 whereas GOLDMERGE-CE emissions only decrease in 2070. In 2100, a gap of 6 GtC/year exists between the two emissions curves. Figures $4 \mathrm{c}$ and $4 \mathrm{~d}$ show the temperature and warming rate trajectories. The warming rate attains the level of $0.2^{\circ} \mathrm{C}$ per decade in 2050 in both models, then the constraint on temperature rate stays active until 2100. Differences for the
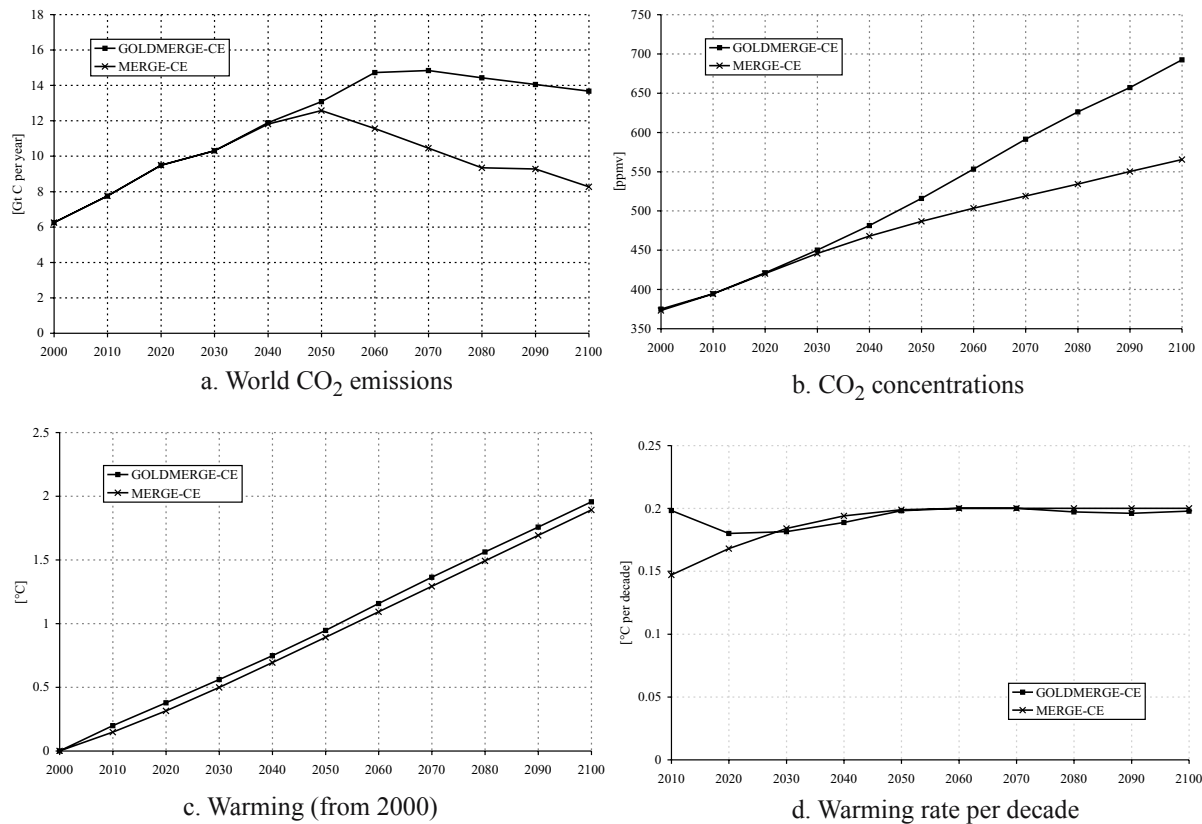

Fig. 4 World energy-related $\mathrm{CO}_{2}$ emissions and atmospheric $\mathrm{CO}_{2}$ concentrations trajectories and warming from 2000 for MERGE in cost-effectiveness mode (MERGE-CE) and GOLDMERGE-CE. 
first period can be explained by the initial climate system inertia which is not the same in the two climate modules. In C-GOLDSTEIN, the initial inertia comes from the initial $\mathrm{CO}_{2}$ forcing used to simulate climate change from the preindustrial era to 2000. In the MERGE climate module, this is parameterised using an additional radiative forcing parameter which defines a lower initial forcing (and hence a lower temperature change of $0.15^{\circ} \mathrm{C}$ in 2010). The cumulative temperatures in Figure $4 \mathrm{c}$ reflect this difference by a small gap between the two curves. In summary, the difference in predicted climate response between the models appears mainly in the difference in the $\mathrm{CO}_{2}$ concentration paths. The gap between the two paths increases with time while the warming rates remain the same due to the constraint. Since the equilibrium climate sensitivities and the warming rates are the same, the larger emissions in GOLDMERGE-CE necessarily imply a larger transient heat uptake by the ocean. Although the ocean lag timescale was chosen to make the response of the two models similar, the behaviour of the more complex model cannot readily be matched by the simple climate module within MERGE. The more realistic climate module is thus allowing larger emissions only to release more heat in the long-term future, beyond the planning horizon. As mentionned in the beginning of the section, the forcing due to other GHGs that are included in MERGE may also be an explanation of the divergence of the two concentration paths.

\subsubsection{GOLDMERGE-CB}

The difference in solution (computation) time between the CE and CB approaches is not significant even though the number of coupling variables is twice as large in the CB case. The coupling variables, temperatures and $\mathrm{CO}_{2}$ concentrations, are plotted in Figure 5. $\mathrm{CO}_{2}$ concentrations are very close in the two models beginning at 373 ppmv in 2000 and reaching 749 ppmv for GOLDMERGE-CB and 745 ppmv for MERGE-CB in 2100. Temperatures have the same dissimilarities described in the previous section. Figure $5 \mathrm{~d}$ details the decadal temperature change rates. In C-GOLDSTEIN the warming rate stays around $0.2^{\circ} \mathrm{C}$ with a slight decrease in 2030 and an increase in the following decades to reach a level of $0.27^{\circ} \mathrm{C}$ in 2100. The evolution of the rate of temperature change in MERGE appears smoother. Energy-related $\mathrm{CO}_{2}$ emissions are plotted in Figure 5a. The two paths are very close, as for the $\mathrm{CO}_{2}$ concentrations paths (Figure 5b), but GOLDMERGE-CB $\mathrm{CO}_{2}$ emissions are slightly lower from 2020 to 2070 then increase quickly after 2070 to exceed the MERGE$\mathrm{CB}$ curve. In summary, the difference in predicted climate response between the models is summarized in the warming rates while the $\mathrm{CO}_{2}$ concentration paths are similar. Interestingly, this is in constrast to the cost-effectiveness approach discussed previously. It may be that in this case the increasing rate of warming permitted by the trade-off between damage and abatement costs is masking the larger potential for long-term heat storage in the more complex model.

\subsection{New perspectives offered by GOLDMERGE}

The experiments with the coupling of the two models MERGE and C-GOLDSTEIN, as described above, show that the method is effective in this extended modelling framework. An interesting opportunity offered by this approach will be to exploit the spatial distribution of the climate variable evolutions provided in the output of C-GOLDSTEIN in order to produce genuine regional damage functions that could influence the economic growth path in the different regions. In Figure 6 we show the spatial distribution of land surface temperature change in 2100 obtained from C-GOLDSTEIN for the case GOLDMERGE-CB. 


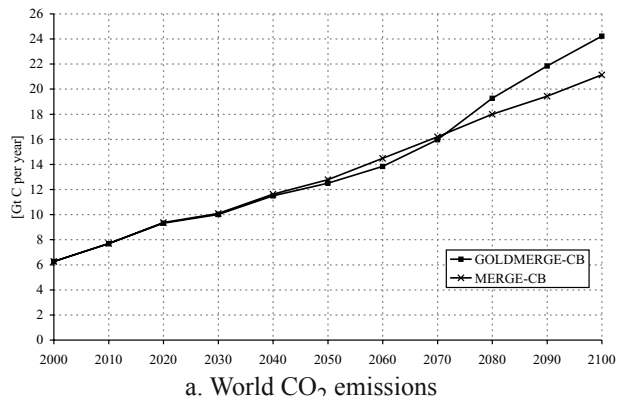

a. World $\mathrm{CO}_{2}$ emissions

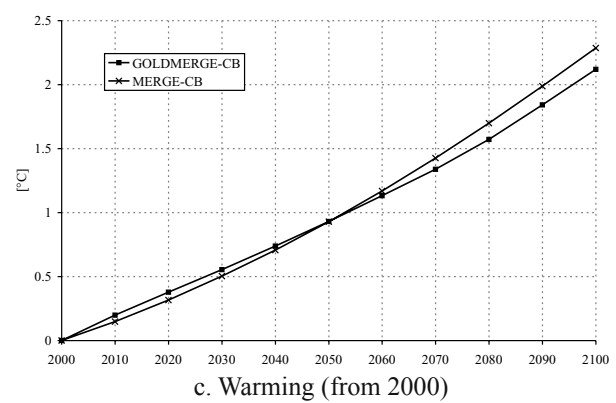

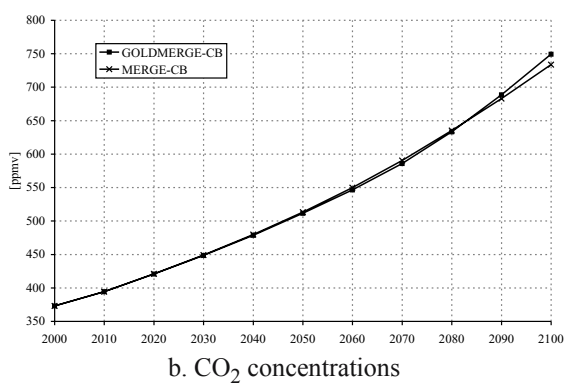

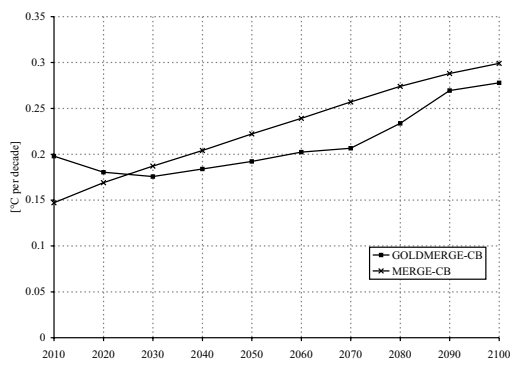

d. Warming rate per decade

Fig. 5 World energy-related $\mathrm{CO}_{2}$ emissions and atmospheric $\mathrm{CO}_{2}$ concentrations trajectories and warming from 2000 for MERGE in cost-benefit mode (MERGE-CB) and GOLDMERGE-CB.
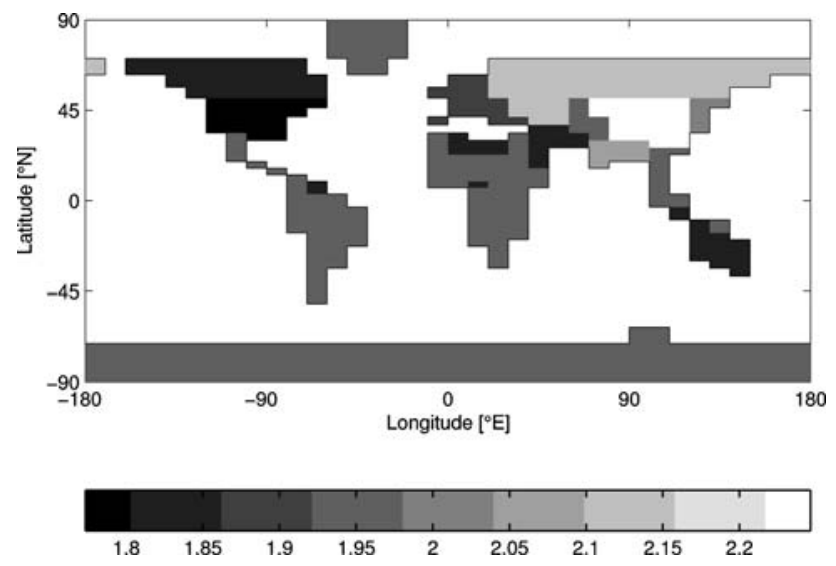

Fig. 6 Distribution of the temperature increase for the standard MERGE regions in 2100 for GOLDMERGECB.

Regionalized temperature increases lie within a range from $1.76^{\circ} \mathrm{C}$ to $2.25^{\circ} \mathrm{C}$. Another important remark concerns the compatibility of the approach with the use of TW constraints. Indeed, since the climate model is sufficiently detailed, the THC shutdown phenomenon is implicitly represented in it. By imposing regionalized "viability" constraints ${ }^{8}$ on purely

\footnotetext{
${ }^{8}$ See (Aubin et al. 2005) for a discussion of viability methods in climate modeling. 
climatic variables like precipitation, temperature, ice and snow cover etc. a tolerable windows approach to climate change policies could be defined based on relatively detailed economic and climate models, while still maintaining computational feasibility. The method could in principle be utilised for the coupling of any optimal economic growth model with any general circulation model. Limitations remain in computation time and model convexity. This kind of coupling is highly relevant for community based assessment because it effectively resolves the problem of the inclusion of complex simulation models inside an optimization framework. To conduct a similar exercise with a high-resolution climate model with a detailed and realistic regional temperature and precipitation response would require significant computational resources, but the knowledge which would be gained may well justify such an investment.

\section{Conclusion}

In this paper we have presented two different ways to establish a dialogue between a climate model and an economic model in order to obtain an integrated assessment of climate policies. First we have shown how a TW constraint can be introduced in the MERGE integrated assessment model in a cost-effectiveness fashion to attempt to prevent the occurrence of a drastic THC weakening. Our TW constraint was obtained from ensemble simulations performed with a spatially resolved climate model (the Bern 2.5-D model) to delineate the conditions that would prevent a THC shutdown. This approach has the advantage of using information coming from a reliable climate model to introduce a TW constraint in MERGE. The climate response to the GHG emissions coming from the economy is however derived from the simplified and schematic climate module that is included in MERGE. In the second example developed here we have described an approach to realize a hard link between an economic growth module and a 3-D climate model of reduced complexity. This approach yields interesting results when the economic growth, carbon cycle and damage modules of MERGE are coupled with the intermediate complexity 3D-model C-GOLDSTEIN. In a reasonable computing time (a few hours on a $2.4 \mathrm{GHz}$ Intel machine) it has been possible to produce consistent simulations of economic growth and climate change paths in either costeffectiveness or cost-benefit analysis frameworks (the GOLDMERGE-CE and -CB models). The tool that has been used to realize the coupling (OBOT) implements an optimization method using "oracles" that correspond to different models.

The results reported here pave the way to the building of more comprehensive and more detailed IAMs. Currently the method is being tested for a coupling between the economic part of MERGE and CBM-GOLDSTEIN (GENIE-1), a more advanced climate model including a description of the carbon cycle. It will also be possible to exploit the fact that the climate model gives a spatially distributed view of the evolution of climate variables. This should permit us to build regional damage functions or to link climate feedbacks with the economic activity (e.g. agriculture). The method could also be extended to realize a coupling between the macroeconomic growth module and a more detailed techno-economic model of the energy sector, in addition to the coupling with a moderate complexity climate model.

Acknowledgements The authors acknowledge financial support by the Natural Sciences and Engineering Research Council of Canada, the Swiss National Science Foundation, and the Swiss National Centre for Competence in Research, NCCR climate. 


\section{References}

Aubin J-P, Bernardo T, Saint-Pierre P (2005) A viability approach to global climate change issues. In: Haurie A and Viguier L (eds.) The coupling of climate and economic dynamics. Kluwer, pp 113-140

Babonneau F, Beltran C, Haurie A, Tadonki C, Vial J-P (2005) Proximal-ACCPM: a versatile oracle based optimization method. Computational and Management Science. Submitted.

Bahn O, Edwards N, Knutti R, Stocker T (2004) Climate policy preserving an atlantic thermohaline circulation collapse. Climatic Change. Submitted. Available in Les Cahiers du GERAD, reference G-2004-72

Bruckner T, Zickfeld K (2004) Low risk emissions corridors for safeguarding the atlantic thermohaline circulation. In: paper presented at the Expert Workshop "Greenhouse Gas Emissions and Abrupt Climate Change", Paris.

Drouet L, Beltran C, Edwards N, Haurie A, Vial J-P, Zachary D (2005a) An oracle method to couple climate and economic dynamics. In: Haurie A and Viguier L (eds.) The coupling of climate and economic dynamics. Kluwer pp 69-94

Drouet L, Edwards N, Haurie (2005b) Coupling climate and economic models in a cost-benefit framework: a convex optimization approach. Environmental Modeling and Assessment. Submitted.

Edwards N, Marsh R (2005) Uncertainties due to transport-parameter sensitivity in an efficient 3-D oceanclimate model. Climate Dynamics 24:415-433

Goffin J-L, Haurie A, Vial J-P (1992) Decomposition and non-differentiable optimization with the projective algorithm. Management Science 38:284-302

Gordon C, Cooper C, Senior C, Banks H, Gregory J, Johns T, Mitchell J, Wood R (2000) The simulation of SST, sea-ice extents and ocean heat transports in a version of the Hadley Centre coupled model without flux adjustments. Climate Dynamics 16:147-168

Haarsma R, Goosse H, Selten F, Opsteegh JD (2001) Decadal variability in high northern latitudes as simulated by an intermediate-complexity climate model. Annals of Glaciology 33:525-532

Hargreaves J, Annan J, Edwards N, Marsh R (2005) Climate forecasting using an intermediate complexity earth system model and the ensemble kalman filter. Climate Dynamics 23(7-8):745-760

IPCC (2001a) Climate change 2001: mitigation, contribution of working group III to the third assessment report of the intergovernmental panel on climate change. Metz B et al. (eds) Cambridge University Press. Cambridge, U.K.

IPCC (2001b) Climate change 2001: the scientific basis, contribution of working group I to the third assessment report of the intergovernmental panel on climate change. Houghtom J et al. (eds) Cambridge University Press, Cambridge, U.K.

Jaeger CC, Leimbach M, Carraro C, Hasselmann K, Hourcade JC, Keeler A, Klein R (2002) Integrated assessment modeling: modules for cooperation. FEEM Working Paper No. 53

Keller K, Bolker B, Bradford D (2004) Uncertain climate thresholds and optimal economic growth. Journal of Environmental Economics and Management 48(1):723-741

Knutti R, Meehl G, Allen MR, Stain forth DA (2005) Constraining climate sensitivity from the seasonal cycle in surface temperature. J. Climate 19:4224-4233

Knutti R, Stocker T (2002) Limited predictability of the future thermohaline circulation close to an instability threshold. Journal of Climate 15:179-186

Knutti R, Stocker T, Joos F, Plattner G-K (2002) Constraints on radiative forcing and future climate change from observations and climate model ensembles. Nature 416:719-723

Knutti R, Stocker TF, Joos F, Plattner G-K (2003) Probabilistic climate change projections using neural networks. Climate Dynamics 21:257-272

Leimbach M, Jaeger C (2004) A modular approach to integrated assessment modeling. Environmental Modelling and Assessment 9(4):207-220

Lenton TM, Williamson MS, Edwards NR, Marsh R, Price AR, Ridgwell AJ, Shepherd JG, and the GENIE team (2005) Millennial timescale carbon cycle and climate change in an efficient Earth system model. Climate Dynamics. Submitted.

Manne A, Mendelsohn R, Richels R (1995) MERGE - a model for evaluating regional and global effects of GHG reduction policies. Energy Policy 23(1):17-34

Manne A, Richels R (2005) MERGE: an integrated assessment model for global climate change. In: Loulou R, Waaub J-P, Zaccour G, (eds) Energy and Environment. GERAD 25th Anniversary Series. Springer, pp $175-189$

Moré J (1983) Recent developments in algorithms and software for trust region methods. In: Mathematical Programming: The State of the Art. Springer Verlag, Berlin, pp. 258-287

Negishi T (1972) General equilibrium theory and international trade. North-Holland.

Nordhaus W (1993) Rolling the 'DICE': an optimal transition path for controlling greenhouse gases. Resource and Energy Economics 15:27-50

Springer 
Nordhaus W, Boyer J (2000) Warming the world: economic models of global warming. MIT Press

Péton O, Vial J-P (2001) A brief tutorial on ACCPM. Technical report. Logilab, University of Geneva

Prinn R, et al. (1999) Integrated global system model for climate policy assessment: feedbacks and sensitivity studies. Climatic Change 3/4(41):469-546

Schmittner A, Stocker T (1999) The stability of the thermohaline circulation in global warming experiments. Journal of Climate 12:1117-1133

Schramm H, Zowe J (1992) A version of the bundle idea for minimizing a non-smooth function: conceptual idea, convergence analysis, numerical results. SIAM Journal on Optimization 2:121-152

Stainforth D, et al (2005) Uncertainty in predictions of the climate response to rising levels of greenhouse gases. Nature 433:403-406

Stocker T, Schmittner A (1997) Influence of $\mathrm{CO}_{2}$ emissions rates on the stability of the thermohaline circulation. Nature 388:862-865

Stocker T, Wright D, Mysak L (1992) A zonally averaged, coupled ocean-atmosphere model for paleoclimate studies. Journal of Climate 4:773-797

Stott P, Mitchell J, Gregory J, Santer B, Meehl G, Delworth T, Allen M (2005) Observational constraints on past attributable warming and predictions of future global warming. J. Climate 19:3055-3069

Toth F, Bruckner T, Fuessel H-M, Leimbach M, Petshel-Held G (2003) Integrated assessment of long-term climate policies: part $1-$ model presentation. Climatic Change 56:37-56

WBGU (2003) Climate protection strategies for the 21st century. Earthscan, London.

Weaver A, Eby M, Wiebe E, Bitz C, Duffy P, Ewen T, Fanning A, Holland M, MacFadyen A, Matthews H, Meissner K, Saenko O, Schmittner A, Wang H, Yoshimori M (2001) The UVic earth system climate model: model description, climatology, and applications to past, present and future climates. Atmos-Ocean 39:361-428 